\title{
Terminology in chronic obstructive lung diseases
}

\author{
C. M. FLETCHER \\ From the University of London
}

SUMmaRY Until the 1960s there was great confusion, both within and between countries, on the meaning of diagnostic terms such as emphysema, asthma, and chronic bronchitis. Proposals made by a group of British doctors in 1959 gradually received widespread acceptance but in recent years some new problems have developed. These include difficulties in the definition of airflow obstruction, recognition that what used to be regarded as a single disease, chronic bronchitis, comprises at least two distinct pathological processes, and uncertainty about the degree of variability which distinguishes asthmatic from more persistent forms of airflow obstruction. These are all problems which could be solved by continuance of appropriate research and of rigorous attention to the principles which determine accurate and acceptable definitions of disease.

Doctors often talk about a disease without saying precisely what they mean. They tend to assume that all other sensible doctors must agree with them. Textbooks of medicine, surgery, and pathology describe the manifestations of disease in detail but seldom attempt precise definitions. They provide diagnostic criteria but their reproducibility, reliability, and intercorrelations are usually assumed, without discussion, to be good. When comparisons have been made of different doctors' diagnoses under carefully controlled conditions, severe inconsistencies between them are almost invariably found (Fletcher and Oldham, 1961).

It is epidemiologists, needing to make accurate comparisons between the frequency and severity of disease in different groups with different exposures to suspected causative agents, who have had to look critically at these aspects of diagnosis. Clinicians are more concerned with stages of disease severe enough to cause troublesome symptoms. This leads them to assume that patients with any disease can be clearly separated from those who appear healthy. A clear example of this fallacy was demonstrated by Cochrane (1965) in relation to a clinical paper in which the ratio of metacarpal length to breadth was advocated as a diagnostic test for Marfan's syndrome. The authors reported a normal distribution of these ratios, but with a sharp break between cases and controls. Cochrane looked at the distribution in a control sample drawn from a general population, and there was no gap. The clinicians had unconsciously rejected all 'borderline' cases in order to demonstrate the discriminatory value of their test. The epidemiologist, avoiding selective prejudice by using a random sample from a general population, was able to demonstrate the fallacy and show that the test was not sharply discriminatory.

A second false but common concept is that diseases are things which have an existence almost independent of the people they affect. This is partly due to the fact that the names of many diseases indicate the agents which cause them. In fact, a disease can be described only by the changes in structure or function which characterise it, and can be defined only in terms of one or more of the manifestations of these changes that are invariably present in every case. Scadding $(1959 ; 1963)$ has emphasised that this characteristic should belong to one, and only one, of four fields of study: aetiology, morbid anatomy, functional disturbance, or clinical features. While the basic definition must be in one of these fields, classification can be based in another field. Thus, for example, tuberculosis is defined in terms of aetiology, but its various forms are classified on the morbid anatomical basis of the organs affected and the pathological changes which occur in them. Diagnostic criteria do not have to be present in every case, but the closer their correlation with the definitive criterion, the greater their value in diagnosis. When a functional disturbance is chosen as the basis for the definition, some limit, often arbitarily chosen but preferably based on prognostic significance, has to be used to give precision to the definition.

\section{DEFINITIONS OF CHRONIC BRONCHITIS} AND EMPHYSEMA

Interest in bronchitis developed in the early 1950s. In the United Kingdom this was partly because of 
the release of chest physicians from concern with tuberculosis after the advent of chemotherapy; also, the introduction of the National Health Service, giving everyone free access to specialist services, brought more patients with bronchitis and emphysema to the attention of consultants, as the poorer sections of the community had always borne the greatest burden of mortality and morbidity from bronchitis. The mortality from this disease has been (and still is) five times as great among unskilled labourers as among those in professional and managerial occupations. It was in 1952 that the Association of Physicians of Great Britain held a symposium on chronic bronchitis at which definition was considered, and Scadding (1952) first suggested that it should be defined in terms of expectoration.

The great London fog of December, 1952, gave a further stimulus to research: 4000 excess cardiorespiratory deaths occurred within a week, and hospital wards were filled with bronchitic patients who had been suddenly precipitated into cardiac and respiratory failure. The Medical Research Council established a Committee for Research into Chronic Bronchitis under the chairmanship of Professor R. V. Christie. It was on this committee that I became aware of Donald Reid's critical interest in all aspects of research in this field. When the committee first met, it was unable to agree on any definition of chronic bronchitis or on the criteria required for its diagnosis. This was especially true of the earlier stages of the disease, since, at that time, 'all but the later stages of the morbid process of chronic bronchitis were poorly understood' (Stuart-Harris and Hanley, 1957). Even in the later stages, there was considerable confusion between the diagnosis of chronic bronchitis and of emphysema. Emphysema was assumed to be present in any patient with severe, persistent airflow obstruction even when no emphysema had been found at autopsy (Postgraduate Medical School, 1951).

\section{IMPROVED DIAGNOSTIC TECHNIQUES}

In order to devise a satisfactory system of definition and classification, one of the first necessities was to develop more accurate and reproducible diagnostic techniques. Work at the Medical Research Council's pneumoconosis research unit had shown that the generally accepted physical signs of emphysema were subject to gross observer error, and correlated poorly with physiological tests (Fletcher, 1952). It is mainly because of this study, and later confirmatory studies, (Godfrey et al., 1969) that epidemiologists have never used physical signs for diagnosis of chest diseases. Clinicians continued to use them in diagnosing emphysema for many years and they still do so, occasionally, as a sort of salutation to traditional clinical expertise of the kind exemplified by the eminent Dr. Samuel Gee (1893), but reliance has steadily come to be placed on physiological methods for accurate diagnosis of emphysema (Fletcher et al., 1963; Burrows et al., 1966). Clinical histories were also found to be unreliable when Cochrane et al. (1951) showed that simple clinical questions about cough, sputum, and breathlessness, applied by different doctors to samples from a population of miners, could produce prevalences which varied threefold. To overcome this, a standardised questionnaire on respiratory symptoms was developed. Its validity and reproducibility were tested in a duplicate survey of Post Office workers in London (Fletcher et al., 1959) for which Donald Reid provided continual encouragement and help. Although considerable observer and subject error were still present, bias could be reduced to a degree which made the method suitable for epidemiological comparisons (Fairbairn et al., 1959). Another method proposed for quantitative assessment of hypersecretion was to measure sputum produced in the first hour after rising. This appeared to be a more quantitative method than asking questions but it has now been shown to be a less valid measure (Fletcher et al., 1974). The questionnaire was later accepted and published by the Medical Research Council's Bronchitis Research Committee, and it has received wide international recognition and use (Medical Research Council, 1976). At the same time, pathologists were developing techniques pioneered by Professor Gough in Cardiff for reproducible assessment of the severity of emphysema (Medical Research Council, 1975), which has facilitated a number of studies of its prevalence and pathogenesis (Thurlbeck, 1976).

In the 1950 s, before these techniques had been developed, there were still major international disagreements in diagnosis.

One of the striking features of mortality statistics at that time was the enormously greater death rate from bronchitis and emphysema in the United Kingdom than in any other country. Donald Reid was for many years concerned with studying the reality and nature of these international differences. The first step was to discover whether the differences implied by recorded death rates were real. They could be due simply to different customs of death certification or to differences in doctors' diagnostic habits. Reid and Rose (1964) developed a systematic approach by presenting to groups of some 30 hospital registrars in London, Bergen, and Boston, the case histories, and, where possible, autopsy reports, of a series of patients who had died in British hospitals from a wide range of cardiovascular 
and respiratory diseases. They were asked to 'certify' the underlying cause of death in these patients according to their own conventions and habits. When these 'death certificates' were coded, great differences were found. The death of a patient classified by the British as due to 'chronic bronchitis' tended to be classified as 'bronchiectasis' or as 'emphysema' by the Norwegian and American physicians.

\section{THE CIBA AND MEDICAL RESEARCH}

COUNCIL PROPOSALS

In 1957, when I visited a number of Departments of Respiratory Medicine in the United States of America, it became clear that the hospital patient who would be generally described as having chronic bronchitis in England was almost always described as having emphysema in America. One reason for this was a traditional belief among American clincians that bronchitis was an unimportant and trivial condition, the concern of general practitioners rather than of specialists. Emphysema tended to be defined in terms of an increase in the RV/TLC ratio. Since patients with asthma usually share this abnormality, they were often diagnosed as having emphysema which could come and go with the onset and remission of their attacks. On my return, I consulted some colleagues, and the Ciba Foundation gave us the opportunity to hold a three-day discussion of the whole diagnostic problem (Ciba Foundation, 1959). The first decision reached by this group of chest physicians and pathologists was that emphysema should be defined on a morbid anatomical basis as the enlargement of the size of air spaces in the lung peripheral to terminal bronchioles. Donald Reid suggested that we needed some general term to include emphysema, asthma, and bronchitis. He proposed 'chronic non-specific lung disease' and the term was accepted. It has been widely used, but it is cumbersome, and its definition requires the exclusion of a number of other cardiac and chest conditions which may cause expectoration and dyspnoea.

Within this group, it was suggested that patients should be classified according to three types of respiratory disorder: (1) Chronic or recurrent excessive secretion of bronchial mucus (chronic bronchitis), which was to be diagnosed by standardised questions on phlegm production; (2) Intermittent obstruction to bronchial airflow (asthma); and (3) Persistent obstruction to bronchial airflow due either to emphysema, as already defined, or to an obstructive form of bronchitis for which the anatomical basis had not yet been discovered. The term 'irreversible obstructive lung disease' was proposed to describe patients with combined emphysema and chronic bronchitis. It is interesting in retrospect to point out that the word 'disease' in the term is superfluous. These patients could more simply be described as having irreversible airflow obstruction.

These new proposals soon received international recognition (World Health Organisation, 1961; American Thoracic Society, 1962; Orie and Sluiter, 1964). Nevertheless, some confusion remained. The term 'chronic bronchitis' continued to be used without qualification to indicate, at one extreme, regular production of small quantities of sputum without any abnormality of lung fuction, and, at the other extreme, severe airflow obstruction associated with expectoration. The use of a single term to cover such a wide range of abnormalities was justified by the hypothesis that they embraced the natural course of a single disease, and that it was important for preventive purposes to identify potentially serious chronic bronchitis at what was regarded as its earliest stage, a mere productive cough (Fletcher et al., 1959; Mitchell, 1969). This concept of a single bronchitic disease was reinforced by a report from the Medical Research Council's Committee on Research into Chronic Bronchitis (1965), which proposed that the disease should be classified into three stages (simple, mucopurulent, and obstructive) for which definitions were given. Standardised ways of measuring the severity of airflow obstruction by simple spirometric methods were also proposed.

A leading article in the Lancet (1965) commented favourably on this report as follows: 'Some may fear that a report of an official body such as this will inhibit original thought and research by the imposition of agreement. This seems unlikely. There can be no doubt that the simple, mucopurulent and obstructive forms of chronic bronchitis described in the report exist, though their interrelationship in the natural history of the disease remains uncertain. Agreement, at least for the time being, on the use of terms is likely to facilitate comparisons between the investigations of researchworkers in different units; the discipline of clear thought about the meaning of diagnostic terms is likely to clarify, not obscure, realities'. In spite of the warning of uncertainty in the inter-relationships between the three components, many people wrongly assumed that the report did suggest that airflow obstruction in 'obstructive bronchitis' was due to obstruction of the airways by 'bronchitis', that is, by mucus gland hypertrophy and by intraluminal mucus (Thurlbeck et al., 1970).

Another possible pathological basis for the definition of chronic bronchitis was developed when Lynne Reid (1960) proposed a quantitative measure- 
ment of mucous gland enlargement in terms of gland/wall thickness ratio, the so-called Reid index. This index has been widely used by pathologists in spite of various technical difficulties, such as obliquely cut bronchi and local variations in glandular tissue, and a number of modifications have been proposed. It has not been used for definitive purposes in epidemiological or clinical studies because it can be measured only at necropsy, and correlations between the index and antemortem assessment of expectoration have not been close (Thurlbeck, 1976).

\section{SUBSEQUENT DEVELOPMENTS}

For about 10 years after the publication of the Ciba report there appeared to be growing international agreement on definitions of chronic non-specific lung diseases. But with further experience and with advancing knowledge some problems and difficulties have arisen.

\section{CHRONIC NON-SPECIFIC LUNG DISEASE}

Several new terms have been introduced, chiefly intended to give greater emphasis to the cause of disability in this group of diseases. The most widely used is 'chronic obstructive lung disease' (COLD), occasionally modified, by people who prefer longer words, to 'chronic obstructive pulmonary disease'. An important difficulty here is that airways obstruction is a difficult concept to define (Macklem, 1971). There may be considerable obstruction in peripheral airways without any measurable change in total resistance to airflow. In emphysema the obstruction may be present only on expiration, and is chiefly due to loss of lung elasticity, allowing airways that are structurally normal to collapse. For these reasons it is best to describe the abnormality as chronic airflow obstruction, and, at least for epidemiological purposes, to define it in terms of a defined degree of abnormality of simple tests of expiratory airflow, such as the Forced Expiratory Volume in one second $\left(F E V_{1}\right)$, or the Peak Expiratory Flow Rate (PEFR). In the absence of general agreement on what degree of loss should be regarded as indicating 'disease', it is necessary for each investigator to decide and state what limits he intends to use to indicate 'obstruction'. The problem here is the same as that encountered in any other disease defined on a functional basis, such as hypertension or diabetes. There, too, a largely arbitary decision has to be taken on where, in the whole distribution of values, the upper or lower limits of 'normal' are to be set. Most epidemiologists have avoided this problem by simply recording differences in mean test values between groups of subjects. One objection to this custom, in relation to causative factors such as smoking, is that only a minority of smokers appear to be sufficiently susceptible to smoking to develop significant airflow obstruction. Thus, with ageing, the distribution of FEV levels or of severity of emphysema at autopsy becomes skewed, as a majority of subjects remain normal but there develops a 'tail' of clinically significant abnormalities. The mean value of a group or population with such a distribution is not the best index for comparing the effects of smoking or any other agent; for the effect on the susceptible minority tends to be overwhelmed by the unaffected majority (Fletcher et al., 1976).

\section{EMPHYSEMA}

The Ciba Group's recommendation that emphysema should be defined on a pathological basis has been universally accepted, with the additional criterion that destructive changes in the alveoli are a necessary definitive characteristic (World Health Organisation, 1961). To the three main types of emphysema, panlobular, centrilobular and irregular, a fourth, paraseptal emphysema, has now been added. Differences in the clinical and functional significance of these four different types of emphysema have not yet been determined (Thurlbeck, 1976).

\section{SMALL AIR WAYS DISEASE}

It used always to be assumed that the airflow obstruction of a patient with 'chronic obstructive bronchitis' was due to an associated emphysema. But reports began to appear (Postgraduate Medical School, 1951; Depierre et al., 1972; Matsuba and Thurlbeck, 1973) of patients dying of severe airflow obstruction with little or no evidence of emphysema at autopsy but with extensive inflammatory obstruction and obliteration of small bronchioles. The term 'small airways disease', first used by Hogg et al. (1968), has come to be applied to this condition.

\section{CHRONIC BRONCHITIS}

Many epidemiological studies have used the Medical Research Council's questionnaire, or others directly derived from or comparable to it (Medical Research Council, 1976). These studies would have been impossible without a standardised method of data collection. They have not only permitted comparisons to be made between the prevalence of individual symptoms in different populations, but they have also compared the prevalence of groups of symptoms. This has been done because it was thought that simple bronchitis-mere chronic expectoration-was too mild a condition to carry much weight in respect of morbidity and mortality. A common grouping, used by Donald Reid in many 
studies and called 'chronic bronchitis', has been 'persistent cough and phlegm lasting three weeks or more plus shortness of breath while walking with other people at an ordinary pace' (Reid et al., 1966).

This emphasis on the symptoms of cough and phlegm was based on the belief that smokers (or others) who developed them were those who would later become disabled by airflow obstruction. Indeed, follow-up of groups with these symptoms does show that they have a higher death rate from cardiorespiratory diseases than those who are free from these symptoms (Krueger et al., 1970). It was not realised that after standardisation for the symptom of breathlessness or FEv level (Higgins and Keller, 1970), the increased risk of people with productive cough disappeared. The results of an eight-year follow-up in Britain (Fletcher et al., 1976; Fletcher and Peto, 1977) and of a twelve-year follow-up in France (Kauffman et al., 1979) indicate that mucous hypersecretion, with or without bronchial infection, and airflow obstruction are, in fact, two largely distinct morbid processes caused by linked susceptibilities to smoking and other forms of air pollution. Thus there is a hypersecretory disorder, corresponding to simple and mucopurulent bronchitis, and a distinct obstructive disorder, due in varying proportions to small airways disease and emphysema in which mucous hypersecretion and clinical bronchial infection play no causal role.

Recognition of the distinction between these two pathological processes requires reconsideration of the definition of the term 'chronic bronchitis'. If this term had continued to be used to indicate only expectoration, its meaning would have remained clear and acceptable, but its use to describe various symptomatic and clinical syndromes is now confusing and misleading. Clinicians still use it, especially in the United Kingdom, in many different senses, ranging from simple expectoration, without disablement, to fatal respiratory failure. It is even used to include the expectoration commonly found in patients with asthma. The uselessness of the word has been finally demonstrated by a wHO expert committee on the epidemiology of chronic nonspecific respiratory diseases (World Health Organisation, 1975) which proposed the definition: 'A non-neoplastic disorder of structure or function of the bronchi usually resulting from prolonged or recurrent exposure to infectious or non-infectious irritation'. This definition not only breaks the principle that definitions of diseases must always be based on characteristics that are present in every case, but it is so comprehensive that it includes all patients with asthma and bronchiectasis, many with chronic heart failure or with viral infections of the bronchi, and those with rare diseases such as bronchial amyloidosis or sarcoidosis. It is remarkable how absurd the conclusions of committees even of intelligent people may sometimes be if they ignore important principles. This wHO definition would, if anyone took notice of it, make confusion worse confounded in the epidemiology of chronic respiratory disease.

Fletcher et al. (1976) suggested that the word 'bronchitis' should be used as little as possible, and that in its place the different components which have been regarded as contributing to the disease to varying extents should be specifically named in any publication or discussion of them. For this purpose the terms mucous hypersecretion (or 'bronchial catarrh', to revert to Laennec's simple terminology); bronchial infection (together with the diagnostic criteria used in any particular study); and irreversible airflow obstruction (or its components, intrinsic airways disease and emphysema) would suffice.

\section{ASTHMA}

The definition proposed by the Ciba Group in 1959 was as follows: 'Asthma refers to the condition of subjects with widespread narrowing of the bronchial airways which changes its severity over short periods of time either spontaneously or under treatment and is not due to cardiovascular disease'. This definition has been widely accepted in principle, but it has not been of much practical value, because the airflow obstruction caused by emphysema and small airways disease is also to some extent reversible, and it has not been possible to define, either in absolute or proportionate terms, the degree of variability that should be accepted as characterising asthma.

The working group which met after the 1971 Ciba meeting concluded that any definition must at present be based on functional abnormality. Some participants had stongly advocated a clinical definition based on a history of episodic dyspnoea, but this is excluded by the lack of any standard method for obtaining such a history. Moreover, some asthmatic patients have continual, not episodic, dyspneoa. A pathological basis should be possible but too little is at present known about lung pathology in mild asthma. Aetiology could be used as a basis in cases caused by type 1 allergy, but there are many patients in which there is no evidence of this abnormality. A functional basis is logical because obstruction to airflow is an invariable characteristic of all patients with asthma, at least when the disease is active, and it is an abnormality which can readily be measured by simple means.

In 1973 the Medical Research Council's bronchitis committee started a study of the distribution of variability of airflow obstruction in patients with 
various types of obstruction and in subjects discovered in field surveys to have evidence of obstruction (Burr et al., 1975). The purpose was to see whether there were separable groups with different degrees of reversibility or variability, whether these characteristics showed a continuous distribution, with the asthmatic types at one end and the bronchitic and emphysematous types at the other, and whether there were associated characteristics, such as eosinophilia of blood or sputum, which could be used as diagnostic criteria. This study was showing some promise, with interesting patterns emerging, when the Medical Research Council decided to disband its bronchitis committee and the study was brought to an end.

Meanwhile, the recommendation of the Ciba working group (Ciba Foundation, 1971) remains valid. This recommendation stated that 'confusion could be reduced if all those who used the word asthma in publications were to provide as much detailed information as possible on symptoms and signs including characteristics of the sputum, on tests of lung function (including blood gases) on precipitating factors, on evidence of immunological abnormalities, on bronchial hyperreactivity and where appropriate on anatomical changes'. It is certain that a classification of asthma under the original Ciba definition could distinguish some clearly definable groups based on the presence or absence of an increase in IgE immunoglobulins and on specific allergic causative factors. The most difficult group to define is the so-called late onset, intrinsic asthma in which no specific immunological abnormality has yet been identified, and which may be difficult to distinguish from other types of chronic airflow obstruction.

\section{Conclusions}

It is sad that there is no longer such wide agreement on the definitions and classification of chronic non-specific lung diseases as appeared to have been reached in the 1960s, but much of value remains and the outlook is still favourable. The word 'emphysema' does now have the same meaning to nearly all clinicians, radiologists, and pathologists. The only persistent disagreement is about whether simple dilation of the alveoli without destruction can be regarded as a form of emphysema, as Reid (1960) proposed. It would seem simple and logical in such cases to use the term 'alveolar dilation' to avoid confusion with destructive emphysema.

With chronic bronchitis, the main problem is to change set habits. There is no real problem in defining or quantifying expectoration, frequency of chest illnesses, or the presence and severity of airflow obstruction. It is necessary, somehow, to discourage people from using the words "chronic bronchitis' except in relation to simple expectoration, and to hope that French influence may gradually be brought to bear upon doctors to return to using the simple term 'bronchial catarrh' introduced by Laennec. In relation to asthma, it is perhaps best to encourage the continuing use of the Ciba definition (Ciba Foundation, 1959) until advances in knowledge enable us to use a full aetiological definition and classification.

It is difficult to overestimate the contributions made to this whole subject by Donald Reid, by his demonstration in the 1960s of the confusion brought about by intra- and international differences in both the definition and the diagnosis of obstructive lung diseases, and by his enthusiastic support for, and continual wise guidance and advice to, all those who were working towards greater consistency and understanding. If, as I believe, epidemiologists have been chiefly responsible for the advances that have been made, Donald Reid must be given pride of place among them.

Reprints from Professor Charles Fletcher, 20 Drayton Gardens, London SW10 9SA.

\section{References}

American Thoracic Society (1962). Chronic bronchitis, asthma and pulmonary emphysema: A statement by the Committee on Diagnostic Standards for Nontuberculous Respiratory Disease. American Review of Respiratory Disease, 85, 762-768.

Burr, M. L., St. Leger, A. S., Bevan, C., and Merrett, T. G. (1975). A community survey of asthmatic characteristics. Thorax, 30, 663-668.

Burrows, B., Fletcher, C. M., Heard, B. E., Jones, N. L., and Wootliff, J. S. (1966). The emphysematous and bronchial types of airway obstruction. A clinicopathological study of patients in London and Chicago. Lancet, 1, 830-835.

Ciba Foundation Guest Symposium (1959). Terminology, definitions and classification of chronic pulmonary emphysema and related conditions. Thorax, 14, 286-299.

Ciba Foundation Study Group No. 38 (1971). Identification of Asthma. Churchill Livingstone: Edinburgh and London.

Cochrane, A. L. (1965). Science and syndromes. Postgraduate Medical Journal, 41, 440-442.

Cochrane, A. L., Chapman, P. J., and Oldham, P. D. (1951). Observers' errors in taking medical histories. Lancet, 1, 1007-1011.

Depierre, A., Bignon, J., Lebeau, A., and Brouet, G. (1972). Quantitative study of parenchyma and small conductive airways in chronic non-specific lung disease. Chest, 62, 699-712.

Fairbairn, A. S., Wood, C. H., and Fletcher, C. M. (1959). Variability in answers to a questionnaire on respiratory symptoms. British Journal of Preventive and Social Medicine, 13, 175-182. 
Fletcher, C. M. (1952). The clinical diagnosis of pulmonary emphysema - an experimental study. Proceedings of the Royal Society of Medicine, 45, 577-582.

Fletcher, C. M., and Oldham, P. D. (1961). Bibliography on observer error and variation. In Medical Surveys and Clinical Trials, pp. 43-49. Edited by L. J. Witts. Oxford University Press: London.

Fletcher, C. M., and Peto, R. (1977). The natural history of chronic airflow obstruction. British Medical Journal, 1, 1645-1648.

Fletcher, C. M., Elmes, P. C., Fairbairn, A. S., and Wood, C. H. (1959). The significance of respiratory symptoms and the diagnosis of chronic bronchitis in a working population. British Medical Journal, 2, 257-266.

Fletcher, C. M., Hugh-Jones, P., McNicol, M. W., and Pride, N. B. (1963). The diagnosis of pulmonary emphysema in the presence of chronic bronchitis. Quarterly Journal of Medicine, 32, 33-49.

Fletcher, C. M., Peto, R., and Tinker, C. M. (1974). A comparison of the assessment of simple bronchitis (chronic mucus hypersecretion) by measurement of sputum volume and by standardised questions on phlegm production. International Journal of Epidemiology, 3, 315-320.

Fletcher, C. M., Peto, R., Tinker, C. M., and Speizer, F. S. (1976). The Natural History of Chronic Bronchitis and Emphysema. Oxford University Press: London.

Gee, S. (1893). Auscultation and Percussion: together with other methods of physical examination of the chest. Fourth edition. Smith Elder and Co: London.

Godfrey, S., Edwards, R. H. T., Campbell, E. J. M., Armitage, P., and Oppenheimer, E. A. (1969). Repeatability of physical signs in airways obstruction. Thorax, 24, 4-9.

Higgins, M. W., and Keller, J. B. (1970). Predictors of mortality in the adult population of Tecumseh. Respiratory symptoms, chronic respiratory disease and ventilatory lung function. Archives of Environmental Health, 21, 418-423.

Hogg, J. C., Macklem, P. T., and Thurlbeck, W. M. (1968). Site and nature of airway obstruction in chronic obstructive lung disease. New England Journal of Medicine, 276, 1355-1360.

Kauffman, F., Drouet, D., Lellouch, J., and Brille, D. (1979). International Journal of Epidemiology. (In press).

Krueger, D. E., Rogot, E., Blackwelder, W.C., and Reid, D. D. (1970). The predictive value of a postal questionnaire on cardiorespiratory symptoms. Journal of Chronic Diseases, 23, 411-421.

Lancet (1965). Leading article: Classification of chronic bronchitis. Lancet, 1, 793.

Macklem, P. T. (1971). Airway obstruction and collateral ventilation. Physiological Reviews, 51, 368-436.

Matsuba, K., and Thurlbeck, W. M. (1973). Disease of the small airways in chronic bronchitis. American Review of Respiratory Disease, 107, 552-557.

Medical Research Council (1965). Definition and classification of chronic bronchitis for clinical and epidemiological purposes. A report to the Medical Research Council by their committee on the aetiology of chronic bronchitis. Lancet, 1, 775-779.
Medical Research Council (1975). Quantitative assessment of chronic non-specific lung disease at necropsy. Report by a panel of the Medical Research Council Committee on Research into Chronic Bronchitis. Thorax, 30, 241-252.

Medical Research Council (1976). Questionnaire on Respiratory Symptoms and Instructions for Interviewers. Medical Research Council: London.

Mitchell, R. S. (1969). Outlook in emphysema and chronic bronchitis. New England Journal of Medicine. 280, 445-452.

Orie, N. G. M., and Sluiter, H. S. (1964). Editors of Bronchitis II, Second International Symposium 22-24 April, 1964, University of Groningen, the Netherlands. Royal Vangorum: Assem and Charles C. Thomas: Illinois.

Postgraduate Medical School (1951). Emphysema, A report of a clinicopathological conference. Postgraduate Medical Journal, 27, 25-35.

Reid, D. D., and Rose, G. A. (1964). Assessing the comparability of mortality statistics. British Medical Journal, 2, 1437-1439.

Reid, D. D., Cornfield, J., Markus, R. E., Seigel, D., Pedersen, E., and Haenszel, W. (1966). Studies of disease among migrants and native populations in Great Britain, Norway and the United States. III. Prevalence of cardiorespiratory symptoms among migrants and native born in the United States. National Cancer Institute Monograph No. 17, pp. 321-346.

Reid, L. (1960). Measurement of the bronchial mucous gland layer. A diagnostic yardstick in chronic bronchitis. Thorax, 15, 132-141.

Reid, L. (1967). The Pathology of Emphysema. LloydLuke (Medical Books): London.

Scadding, J. G. (1952). In 'Symposium on Chronic Bronchitis'. Quarterly Journal of Medicine, 21, 460.

Scadding, J. G. (1959). Principles of diagnosis in medicine. Lancet, 1, 323-325.

Scadding, J. G. (1963). Meaning of diagnostic terms in broncho-pulmonary disease. British Medical Journal, 2, 1423-1430.

Stuart-Harris, C. M., and Hanley, T. (1957). Chronic Bronchitis, Emphysema and cor pulmonale. John Wright: Bristol.

Thurlbeck, W. M. (1976). Chronic Airflow Obstruction in Lung Disease. W. B. Saunders and Co: Philadelphia, London, Toronto.

Thurlbeck, W. M., Henderson, J. A., Fraser, R. G., and Bates, D. V. (1970). Chronic obstructive lung disease. A comparison between clinical, roentgenologic functional and morphologic criteria in chronic bronchitis emphysema, asthma and bronchiectasis. Medicine, 49, 81-145.

World Health Organisation (1961). Chronic cor pulmonale: report of an expert committee. Technical Report Series No. 213. WHO: Geneva.

World Health Organisation (1975). Epidemiology of chronic non-specific respiratory diseases. Bulletin of the World Health Organisation, 52, 251-259. 